\title{
Platelet Activity and Phosphoinositide Turnover Increase with Advancing Age
}

EdWARD J. BASTYR, III, M.D., MARK M. KAdROFSKE, B.S., AARON I. VINIK, M.D., Ann Arbor, Michigan

PURPOSE: Blood platelet activity increases with advancing age. This study was designed to determine if changes in a key signal-transducing mechanism in the platelet, phosphoinositide turnover, are associated with the enhanced platelet activity seen in aging.

PATIENTS AND METHODS: Platelets were harvested from a total of 40 healthy, non-obese, 22- to 62year-old individuals, free of any clinical evidence of atherosclerotic vascular disease, and having normal serum laboratory lipid levels. Studies of platelet activity included measurement of in vitro platelet aggregation and plasma $\beta$-thromboglobulin $(\beta$-TBG), a marker of in vivo platelet secretion. Basal and thrombin-stimulated phosphoinositide turnover was measured following [ $\left.{ }^{32} \mathrm{P}\right]$-orthophosphate incorporation into the various phospholipids, isolation of the phosphoinositides and phosphatidic acid by thin-layer chromatography and autoradiography, and quantification by liquid scintillation spectroscopy of these radiolabeled phospholipids. RESULTS: There was a positive correlation with age for both adenosine diphosphate (ADP)-induced aggregation $(1.25 \mu \mathrm{M}, \mathrm{r}=0.464, \mathrm{p}<0.001 ; 2.5 \mu \mathrm{M}, \mathrm{r}$ $=0.386, p<0.05)$ and plasma $\beta$-TBG $(r=0.381, p$ $<0.055)$. There was a time-dependent increase of $\left[{ }^{32} \mathbf{P}\right]$ orthophosphate $\left({ }^{32} \mathbf{P}_{\mathrm{i}}\right)$ incorporation into platelet phosphatidylinositol 4,5-bisphosphate $\left(\mathrm{PIP}_{2}\right)$ and phosphatidylinositol 4-phosphate (PIP), and isotopic equilibrium was reached by 120 minutes at $37^{\circ} \mathrm{C}$. A positive correlation was found between age and basal ${ }^{32 P}$-PIP $2(r=0.640, p<0.001)$ and ${ }^{32}$ P-PIP $(r=0.676, p<0.0005)$. Basal ${ }^{32} P_{i}$ incorporation into PIP $_{2}$ correlated positively with in vitro aggregation $(1.25 \mu \mathrm{M}$ ADP, $\mathrm{r}=0.795, \mathrm{p}<0.0001 ; 2.5 \mu \mathrm{M}$ ADP, $\mathrm{r}=$ $0.755, p<0.0005)$ as did ${ }^{32} P_{i}$ incorporation into PIP $(1.25 \mu \mathrm{M}$ ADP, $\mathrm{r}=0.815, \mathrm{p}<0.0001 ; 2.5 \mu \mathrm{M}$ ADP, $\mathrm{r}=$ 0.795 , p < 0.0001 ). There was also a positive correlation between plasma $\beta$-TBG levels and basal ${ }^{32} \mathbf{P}$ $\operatorname{PIP}_{2}(r=0.768, p<0.005)$ and ${ }^{32 P}$-PIP $(r=0.505, p$ $<\mathbf{0 . 0 6 6}$ ). Finally, increasing age correlated with thrombin $(4 \mathrm{U} / \mathrm{mL})$-stimulated ${ }^{32}$ P-PIP 2 hydrolysis $(r=0.694, p<0.01)$ and phosphatidic acid formation $(\mathbf{r}=0.556, p<0.05)$.

\footnotetext{
From the Department of Internal Medicine, Division of Endocrinology and Metabolism, and the Department of Surgery, University of Michigan, Ann Arbor, Michigan. This work was supported by grants from the National Institute of Diabetes and Digestive and Kidney Diseases of the National Institutes of Health (1F32DK08108-01), the Michigan Diabetes Research and Training Center (2P60DK20572-11), and Roerig, Division of Pfizer Inc. This work was presented in part at the meeting of the American Federation of Clinical Research in Washington, D.C., on May 2, 1988. Requests for reprints should be addressed to Aaron I. Vinik, M.D., Department of Internal Medicine and Surgery, 2922B Taubman Center, University of Michigan Medical Center, Ann Arbor, Michigan 48109-0331. Manuscript submitted October 2, 1989, and accepted in revised form February 23, 1990.

Dr. Bastyr's present address is 4.174 Old John Sealy Hospital, University of Texas Medical Branch-Galveston, Galveston, Texas 77550.
}

CONCLUSION: Advancing age is associated with an increase in in vitro platelet aggregation, elevated $\beta$-TBG levels, and enhanced phosphoinositide turnover. Because phosphoinositide turnover is associated with stimulus-coupled platelet activation, the increased basal and stimulated phosphoinositide turnover may mediate the increase in platelet activity with aging.

$A$ $\mathbf{n}$ increase in platelet aggregability has been proposed as a contributing factor to the development of atherosclerotic vascular disease [1]. Advancing age is associated with both atherosclerosis and platelet hyperaggregation [2]. Increased in vitro platelet aggregatory responses to adenosine diphosphate (ADP) with increasing age have been reported [3]. Plasma levels of $\beta$-thromboglobulin ( $\beta$-TBG) (a platelet-specific protein secreted upon activation) increase with age and have been correlated with platelet hyperaggregation [4]. Biochemical mechanisms associated with the in vitro and in vivo increases in platelet activity with aging are not known.

Platelet activation is associated with phosphoinositide turnover and formation of second messengers 1,2diacylglycerol (DAG) and inositol 1,4,5-triphosphate $\left(\mathrm{IP}_{3}\right)$ from hydrolysis of phosphatidylinositol 4,5-bisphosphate $\left(\mathrm{PIP}_{2}\right)$ [5-9]. DAG is rapidly phosphorylated by a DAG kinase to form phosphatidic acid (PA). Calcium mobilization, protein kinase $\mathrm{C}$ activation, and platelet alpha and dense granule secretion are linked to formation of these second messengers $[9,10]$. The impact of aging on phosphoinositide turnover is not known.

In the present study, we have examined the hypothesis that advancing age enhances ADP-induced aggregation and raises plasma $\beta$-TBG as a function of platelet phosphoinositide content and turnover. We confirm here the previous findings that in vitro ADP. induced aggregation and in vivo plasma $\beta$-TBG increase with advancing age. We demonstrate that platelet polyphosphoinositide content correlates with both age and ADP-induced aggregation. Furthermore, upon platelet stimulation with thrombin, $\mathrm{PIP}_{2}$ hydrolysis and PA generation increase as a function of increasing age. We suggest that the increase in in vitro platelet aggregation and raised plasma $\beta$-TBG levels that occur with advancing age are due to an increase in basal and stimulated platelet phosphoinositide turnover.

\section{PATIENTS AND METHODS}

A total of 40 healthy 22 - to 62 -year-old volunteers ( 21 men and 19 women) free of any clinical disease, including evidence of atherosclerotic vascular disease, were recruited for the study. None of the volunteers 
were obese, and all had serum cholesterol, triglyceride, creatinine, and fasting glucose levels within the normal laboratory range. The subjects were instructed to take no medications specifically known to affect platelet function for at least 1 week before the study. Blood sampling was performed at the same early morning hour (8 A.M.) after at least a 12-hour fast. Informed written consent was obtained from each volunteer. The study was approved by the Institutional Review Board Human Use Committee at the University of Michigan Medical Center.

In vitro platelet aggregation was performed by a turbidometric technique similar to that described by Born and Cross [11]. Forty milliliters of whole blood were gently dispersed into a polypropylene tube containing $3.5 \%$ trisodium citrate anticoagulant (9:1, volume:volume [v:v]) and centrifuged in a swinging bucket rotor at $125 \times \mathrm{G}$ for 12 minutes at $22^{\circ} \mathrm{C}$. The platelet-rich plasma (PRP) was removed to within 1 $\mathrm{cm}$ of the buffy coat. The remaining blood was centrifuged at $5,000 \times \mathrm{G}$ for 12 minutes at $22^{\circ} \mathrm{C}$ and the platelet-poor plasma (PPP) removed (less than 100 platelets per $\mu \mathrm{L}$ ). The platelet count was adjusted to $300,000 / \mu \mathrm{L}$ with autologous PPP using an electronic particle counter. Aliquots $(450 \mu \mathrm{L})$ of PRP were incubated at $37^{\circ} \mathrm{C}$ for 2 minutes with constant stirring at 1,200 revolutions/minute and the light transmittance was monitored using an aggregometer. Aggregation was measured on a strip chart recorder in $\mathrm{mV}$ and expressed as a percentage of light transmittance of the PPP. For each aggregation curve, percent aggregation was measured with the light transmittance of PPP representing $0 \%$ and the light transmittance of the PRP representing $100 \%$. Aggregation studies were completed within 3 hours of obtaining samples. Percent aggregation was measured during primary aggregation at 30,60 , and 120 seconds following the addition of 1.25 and $2.5 \mu \mathrm{M}$ ADP. To verify that the platelet release reaction did not occur, adenosine triphosphate (ATP) release from platelet dense-granules was monitored with the luciferin-luciferase reaction by a modification of methods previously described [12].

In vivo platelet activity was determined by measuring plasma levels of $\beta$-TBG, a protein secreted from platelet alpha-granules upon activation [13]. Triplicate $2.5-\mathrm{mL}$ samples of whole blood were placed in plastic tubes on ice containing $150 \mu \mathrm{L}$ of $134 \mathrm{mM}$ EDTA and $15 \mathrm{mM}$ theophylline, and $\beta$-TBG was assayed by radioimmunoassay in the Ligand Laboratory of the Diabetes Research and Training Center at the University of Michigan Medical Center.

Platelet phosphoinositide and PA determinations were carried out by slight modifications of methods established in the literature [14]. Two hundred fifty milliliters of PRP were obtained by plasmapheresis from whole blood collected in an acid/citrate/dextrose anticoagulant buffer ( $71 \mathrm{mM}$ citric acid, $85 \mathrm{mM}$ trisodium citrate, $111 \mathrm{mM}$ dextrose, $\mathrm{pH}$ 5.5, 5:1 v:v). The PRP was centrifuged twice in a swinging bucket rotor at $100 \times \mathrm{G}$ for 12 minutes at $22^{\circ} \mathrm{C}$ to remove any remaining red blood cell contamination. Platelets were pelleted at $1,200 \times \mathrm{G}$ for 10 minutes and suspended in a small volume of incubating buffer $(20 \mathrm{mM}$ TRIS- $\mathrm{HCl}, 150 \mathrm{mM}$ sodium chloride, $5 \mathrm{mM}$ glucose, $0.025 \%$ bovine serum albumin, $\mathrm{pH} 7.4$ ) and brought to $2 \times 10^{9}$ platelets $/ \mathrm{mL}$ with autologous PPP. The final volume of plasma added was $7.5 \pm 0.4 \mathrm{~mL}$ (range of 7.1 to $7.7 \mathrm{~mL}$ ) with a final phosphorus concentration of 0.8 $\pm 0.3 \mathrm{mM}$ (range 0.6 to $1.0 \mathrm{mM}$ ). Aliquots of $500 \mu \mathrm{L}(1$ $\times 10^{9}$ platelets) were incubated with $20 \mu \mathrm{Ci} / \mathrm{mL}$ of carrier-free $\left[{ }^{32} \mathrm{P}\right]$ orthophosphate $\left({ }^{32} \mathrm{P}_{\mathrm{i}}\right)$. After $180 \mathrm{~min}$ utes, the incubation was terminated with the addition of phosphate-washing buffer $(3.2 \mathrm{mM}$ dipotassium phosphate, $24.4 \mathrm{mM}$ monosodium potassium, $4.2 \mathrm{mM}$ disodium phosphate, $150 \mathrm{mM}$ sodium chloride, 5.5 $\mathrm{mM}$ glucose, $0.025 \%$ bovine serum albumin, $\mathrm{pH} 7.4$ ) and then centrifuged at $1,000 \times \mathrm{G}$ for 12 minutes. The wash was repeated, the pellet was resuspended in 500 $\mu \mathrm{L}$ TRIS-HCl pH 7.4 buffer, and the platelet membrane phospholipids were extracted using methanol/ chloroform/hydrochloric acid. The extract was then dried under nitrogen and dissolved in chloroform/ methanol. The extracts were applied to heat-activated oxalate-coated silica plates, and phosphatidylinositol (PI), phosphatidylinositol 4-monophosphate (PIP), $\mathrm{PIP}_{2}$, and PA separated by thin-layer chromatography (TLC) using a Jolles solution [15]. Following TLC and autoradiography, bands were identified and scraped, and the radiolabeled phospholipids were counted by liquid scintillation spectroscopy.

Phosphoinositide turnover and PA formation were also assessed after the addition of thrombin. Aliquots of washed platelets $\left(1 \times 10^{9}\right.$ platelets $)$ were incubated with $20 \mu \mathrm{Ci} / \mathrm{mL}$ of carrier-free ${ }^{32} \mathrm{P}_{\mathrm{i}}$ for 120 minutes at $37^{\circ} \mathrm{C}$. The incubation was terminated with the aforementioned phosphate-washing buffer and then centrifuged at $1,000 \times \mathrm{G}$ for 12 minutes. The platelets were suspended in $500 \mu \mathrm{L}$ of the aforementioned TRIS-HCl $\mathrm{pH} 7.4$ buffer and stimulated with $4 \mathrm{U} / \mathrm{mL}$ human thrombin. The reaction was terminated with $1.0 \mathrm{~mL}$ of cold methanol and chloroform $(2: 1, v: v)$. The radiolabeled phospholipids were extracted, isolated by TLC, and quantitated as just described.

The mass of the individual phosphoinositides was estimated using a spectrophotometric assay for phosphorus determination, as previously described [16]. Briefly, the individual radiolabeled phospholipids were extracted from the silica and dephosphorylated with hot magnesium nitrate, and aliquots were added to a malachite green/ammonium molybdate dye solution. Absorbance was read at $660 \mathrm{~nm}$ in a light spectrophotometer. Recoveries from silica gel were typically as follows: PA 79\%, $\mathrm{PIP}_{2} 39 \%$, PIP $54 \%$, PI 79\%. These are similar to recoveries previously published [17].

Total serum cholesterol, triglycerides, creatinine, and fasting glucose were determined in the Chemical Pathology Laboratory of the University of Michigan Hospital from samples drawn at the time of platelet testing.

All statistical evaluations were performed on the University of Michigan IBM 3090-600E computer. Simple linear regression, multivariate linear regression, and analysis of covariance were performed where appropriate. Coefficients of correlation ( $r$ ) were determined by the method of least mean squares. All data are expressed as mean \pm the standard error of the mean. Statistical significance was accepted at the $95 \%$ confidence level $(p<0.05)$.

\section{RESULTS}

The platelet aggregatory response to both $1.25 \mu \mathrm{M}$ and $2.5 \mu \mathrm{M}$ ADP increased with advancing age (Fig- 


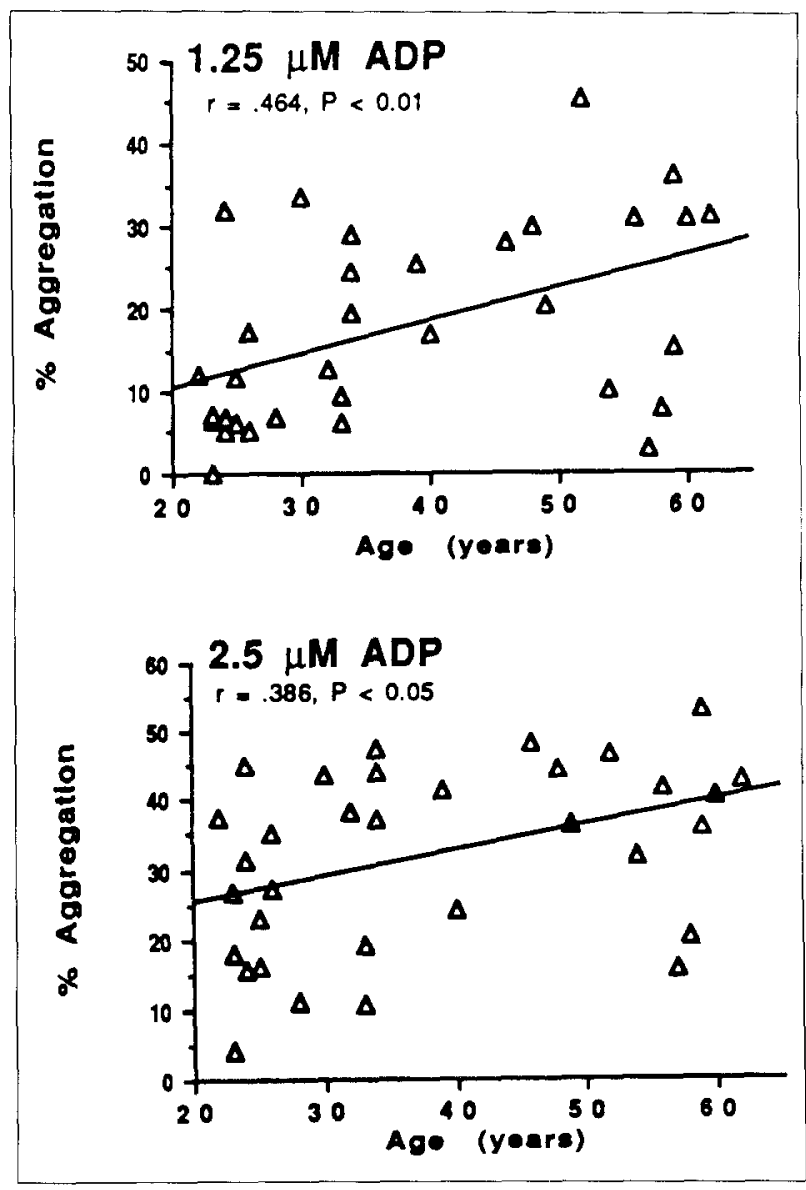

Figure 1. Relationship between in vitro platelet aggregation and age in response to $1.25 \mu \mathrm{M}$ ADP (top) and $2.5 \mu \mathrm{M}$ ADP (bottom). Values $(n=33$ ) for aggregation are given as the mean of percent aggregation measured at 30,60 , and 120 seconds after the addition of ADP. The correlation coefficient for linear regression analysis ( $r$ ) and significance value ( $p$ ) are given.

ure 1). In young subjects the percentage aggregation was $10 \%$ and $25 \%$, respectively, and increased to $30 \%$ and $40 \%$ for the two doses of ADP. There was a significant positive correlation between aggregation and age for both $1.25 \mu \mathrm{M}$ ADP $(\mathrm{r}=0.46, \mathrm{p}<0.01)$ and $2.5 \mu \mathrm{M}$ $\operatorname{ADP}(r=0.386, p<0.05)$. Plasma $\beta$-TBG levels also increased with advancing age. The correlation between plasma $\beta$-TBG and age approached statistical significance $(r=0.381, p<0.055)$. These studies provide evidence for increased in vitro and in vivo platelet activity.

Basal ${ }^{32} \mathrm{P}_{\mathrm{i}}$ incorporation into the phosphoinositides and $\mathrm{PA}$ reached isotopic equilibrium by 120 minutes in every subject tested. In the absence of exogenous agonist, the specific activity ( ${ }^{32} \mathrm{P} / \mu \mathrm{g}$ phosphorus) of $\mathrm{PI}$ and $\mathrm{PIP}_{2}$ reached equilibrium at 60 to 90 minutes for $\mathrm{PIP}_{2}$ and between 90 and 120 minutes for PIP (Figure 2). Thus, labeling of these polyphosphoinositides with ${ }^{32} \mathrm{P}_{\mathrm{i}}$ for 120 minutes reflects their respective phospholipid mass. Therefore, it was possible to examine the relationship between age and mass of the phosphoinositides. There was a significant positive correlation between basal ${ }^{32} \mathbf{P}_{\mathrm{i}}$ incorporation at 120 minutes into PIP and PIP $_{2}$ with advancing age (Figure 3 ). In addition, the incorporation of ${ }^{32} \mathrm{P}_{\mathrm{i}}$ in both phosphoinositides, PIP and $\mathrm{PIP}_{2}$, at the equilibrium time of 120 minutes was found to correlate strongly with the ag-

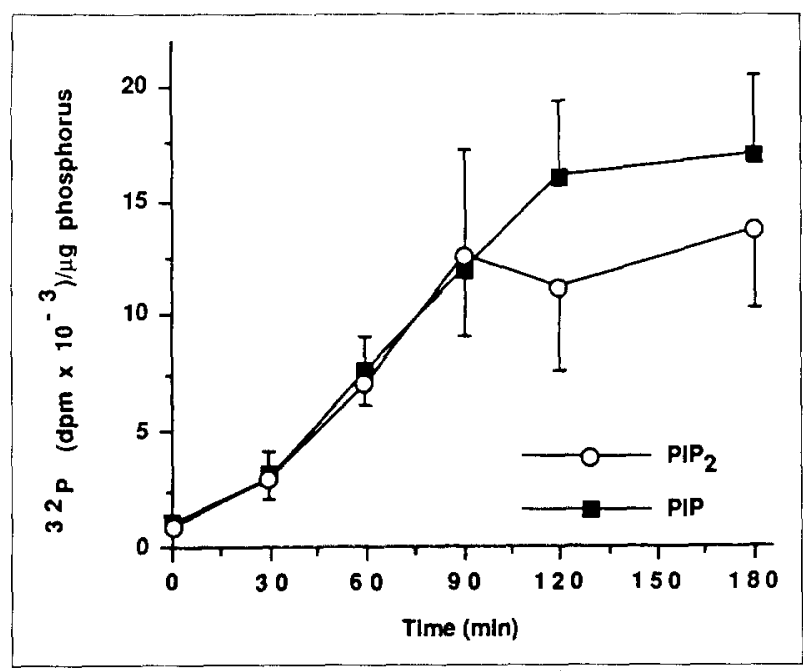

Figure 2. Specific activity of PIP and PIP 2 over 180 minutes Data are represented as ${ }^{32} \mathrm{P}_{\mathrm{i}}$ incorporated per $\mu \mathrm{g}$ phosphorus of the individual polyphosphoinositide. Values are expressed as means \pm standard error from six subjects (one from each decade) determined in duplicate.

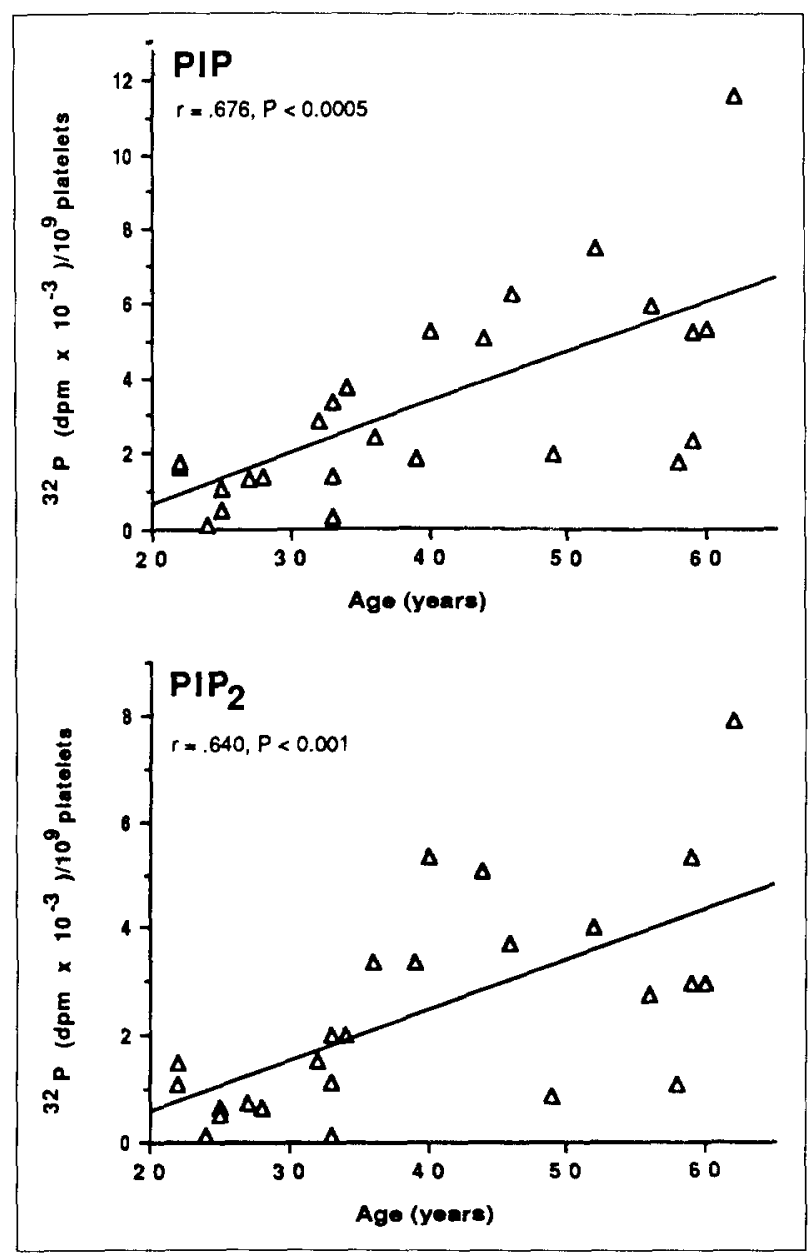

Figure 3. Relationship between ${ }^{32} \mathrm{P}_{\mathrm{i}}$ incorporation into the platelet polyphosphoinositides and age for each individual study subject $(n=25)$. Top, ${ }^{32 P}$-PIP versus age, and bottom, ${ }^{32} \mathrm{P}-\mathrm{PIP}_{2}$ versus age. ${ }^{32} \mathrm{P}_{\mathrm{i}}$ incorporation into the polyphos phoinositides at $37^{\circ} \mathrm{C}$ was measured at 120 minutes (see text). The correlation coefficient for linear regression analysis $(r)$ and significance value $(p)$ are given. 


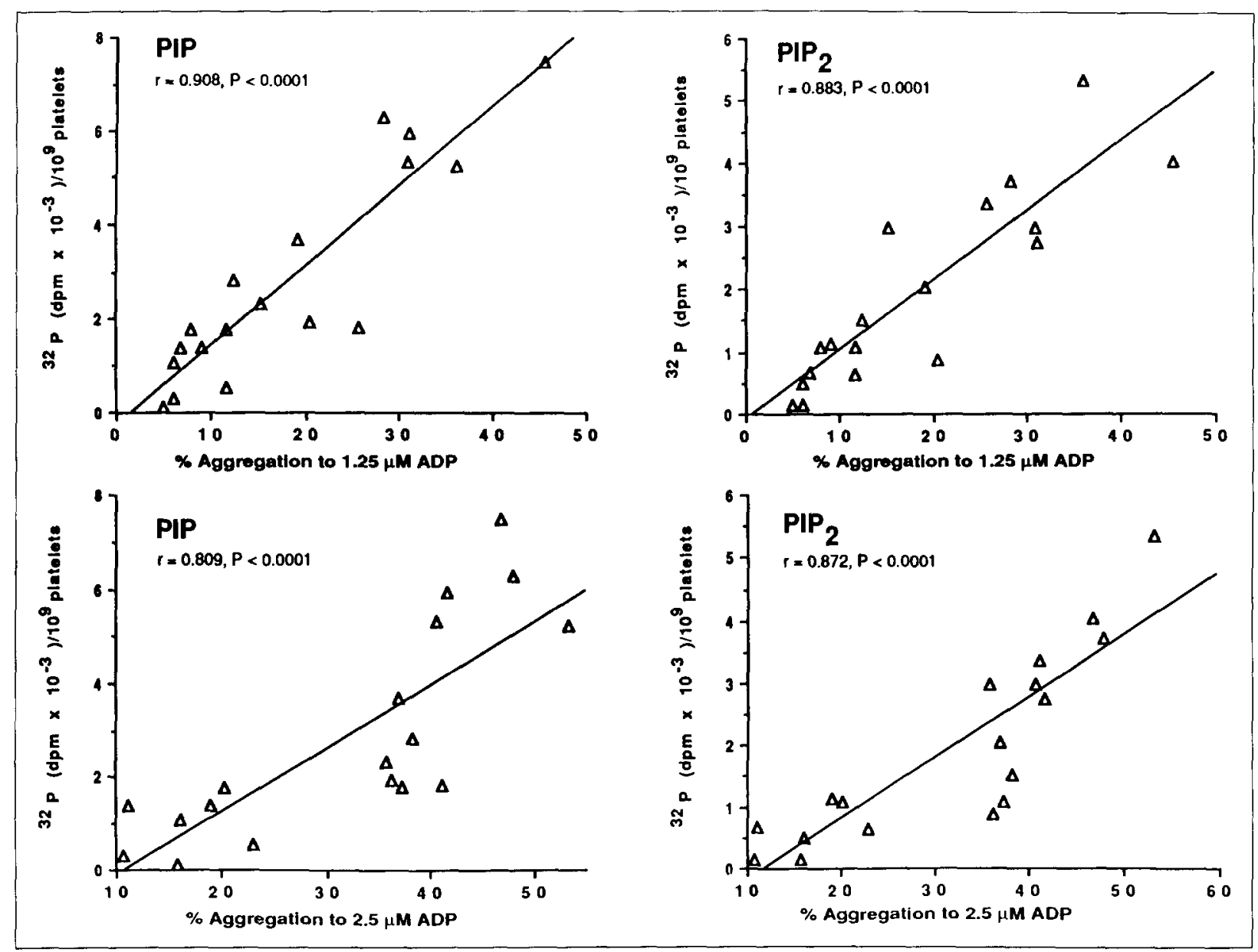

Figure 4. Relationship between platelet aggregation and ${ }^{32} P_{\mathrm{i}}$ incorporation into the platelet polyphosphoinositides $(n=19)$. 32P-PIP versus percent aggregation to $1.25 \mu \mathrm{M} \mathrm{ADP}$ (top left) and $2.5 \mu \mathrm{M} \mathrm{ADP}$ (bottom left), and ${ }^{22} \mathrm{P}-\mathrm{PIP}_{2}$ versus percent aggregation to $1.25 \mu \mathrm{M} \mathrm{ADP}$ (top right) and $2.5 \mu \mathrm{M}$ ADP (bottom right). The correlation coefficient for linear regression analysis ( $r$ ) and significance value $(p)$ are given

gregatory response to both $1.25 \mu \mathrm{M}$ and $2.5 \mu \mathrm{M}$ ADP with correlation coefficients of 0.8 or more and significant levels of $p<0.0001$ (Figure 4). It therefore appeared that in vitro aggregation was a function of phosphoinositide turnover.

Since basal ${ }^{32} \mathrm{P}$ incorporation into the polyphosphoinositides would reflect mass of the compounds and not necessarily turnover, the response to activation with thrombin, which is a potent stimulus of platelet phosphoinositide turnover, platelet aggregation, and platelet secretion, was examined. Platelets from 14 subjects were stimulated with $4 \mathrm{U} / \mathrm{mL}$ human thrombin, which resulted in a time-dependent increase in ${ }^{32} \mathrm{P}-\mathrm{PIP} \mathrm{P}_{2}$ hydrolysis and ${ }^{32} \mathrm{P}-\mathrm{PA}$ generated when the maximum amount of $\mathrm{PIP}_{2}$ hydrolyzed and PA generated was examined. There was an increase in ${ }_{32} \mathrm{P}$-PIP ${ }_{2}$ hydrolysis reflected in decreasing amounts in the $\mathrm{PIP}_{2}$ fraction and increased ${ }^{32} \mathrm{P}$-PA formation that correlated with advancing age (Figure 5).

As a measure of in vivo platelet activation, plasma $\beta$ TBG levels were determined and found to correlate positively with ${ }^{32} \mathrm{P}$ incorporation into both PIP and $\mathrm{PIP}_{2}$, with correlation coefficients of $0.505(p=0.06)$ and $0.768(p<0.005)$, respectively (Figure 6).

Because platelet activation could reflect changes within the platelet per se or asymptomatic atherosclerotic vascular disease, correlates between in vitro and in vivo aggregation and risk factors for vascular disease were sought. No relationship, however, was found between platelet activation and body mass index, total cholesterol, triglycerides, serum creatinine, or fasting glucose.

\section{COMMENTS}

Previous studies have shown that enhanced platelet function is associated with aging in healthy individuals [2-4]. In the present study, we examined the relationship between aging and platelet function and phosphoinositide turnover, a key regulatory mechanism involved in platelet activation. The present data confirm our preliminary findings [18], and those of others [19-21], of increased in vitro platelet aggregation and increased in vivo platelet activity with aging. Furthermore, we demonstrate that the increased platelet activity with aging is associated with increased basal platelet polyphosphoinositide content and increased thrombin-stimulated platelet phosphoinositide turnover. The increased platelet activity with advancing age is independent of metabolic and hemodynamic perturbations such as those seen in diabetes mellitus and hypertension [22-24].

There was a strong positive correlation between age and basal ${ }^{32} \mathrm{P}_{\mathrm{i}}$ incorporation at 120 minutes into $\mathrm{PIP}_{2}$ and $\mathrm{PI}$. In addition, ${ }^{32} \mathrm{P}_{\mathrm{i}}$-labeled polyphosphoinositide 


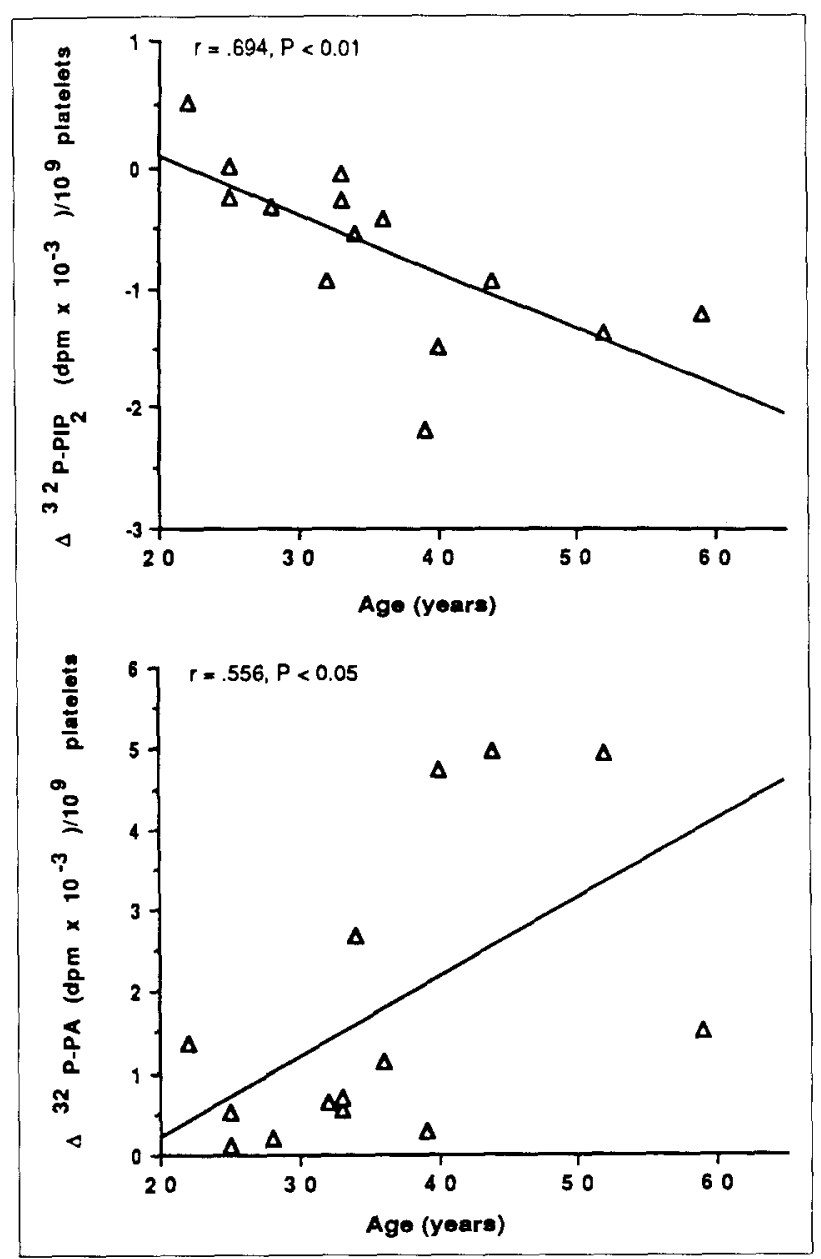

Figure 5. Relationship between $\mathrm{PIP}_{2}$ hydrolysis (top) and PA generation (bottom) and age. Values are expressed $(n=14)$ as change from basal at 15 seconds after platelet stimulation with $4 \mathrm{U} / \mathrm{mL}$ human thrombin. The correlation coefficient for linear regression analysis ( $r$ ) and significance value ( $p$ ) are given.

content correlated positively with in vitro platelet aggregation and plasma $\beta$-TBG release in these same patients. Since ${ }^{32} \mathrm{P}_{\mathrm{i}}$ labeling was found to reach equilibrium in 120 minutes, tracer was taken to reflect mass, suggesting that polyphosphoinositide content was a major factor in the increased in vitro aggregation and the increased in vivo platelet activity associated with aging.

When platelets were stimulated with thrombin, we found a significant positive correlation between age and ${ }^{32} \mathrm{P}-\mathrm{PIP}_{2}$ hydrolysis and ${ }^{32} \mathrm{P}-\mathrm{PA}$ formation. This provides evidence that age-related increased platelet activity may also be associated with an altered transmembrane signaling mechanism involved in the formation of key intraplatelet second messengers. Because basal levels of PIP and $\mathrm{PIP}_{2}$ correlated with thrombin-stimulated levels of ${ }^{32} \mathrm{P}-\mathrm{PIP}_{2}$ and ${ }^{32} \mathrm{P}-\mathrm{PA}$ (data not shown), the stimulated hydrolysis of $\mathrm{PIP}_{2}$ may be a function of basal polyphosphoinositide levels.

The importance of age-related changes in the platelet lipid concentration has been studied previously. Particular reference to the arachidonic acid pathway has been emphasized. Prisco et al [25] have shown that platelet lipid composition in healthy 20 - to 68-year-old subjects had greater concentrations of cholesterol, an increase in the saturated fatty acid content of phosphatidylcholine, and a decrease in the unsaturated fatty acid content with increasing age. These changes have been associated with enhanced aggregability presumably secondary to increased amounts of thromboxane $\mathrm{A}_{2}$ formation [26,27].

Alternatively, decreased levels of linoleic acid and unsaturated fatty acids in the platelet have been implicated as a causal mechanism for increased platelet hypersensitivity in the elderly [28]. Prostaglandin synthesis and cyclooxygenase activity are inhibited by unsaturated fatty acids [29]. It is known that increased thromboxane $\mathbf{A}_{2}$ production and decreased prostacyclin formation occur with advancing age. If arachidonate metabolites were primary to the increased platelet aggregation in aging, it might be expected that inhibition of these pathways in vivo would alter clinical outcome. However, this has not been the case. In the Veterans Administration Cooperative Study, the amputation rate in a group of individuals with peripheral vascular disease receiving aspirin was not different from that in patients treated with placebo [30]. Hence, formation of arachidonate metabolites may not be the

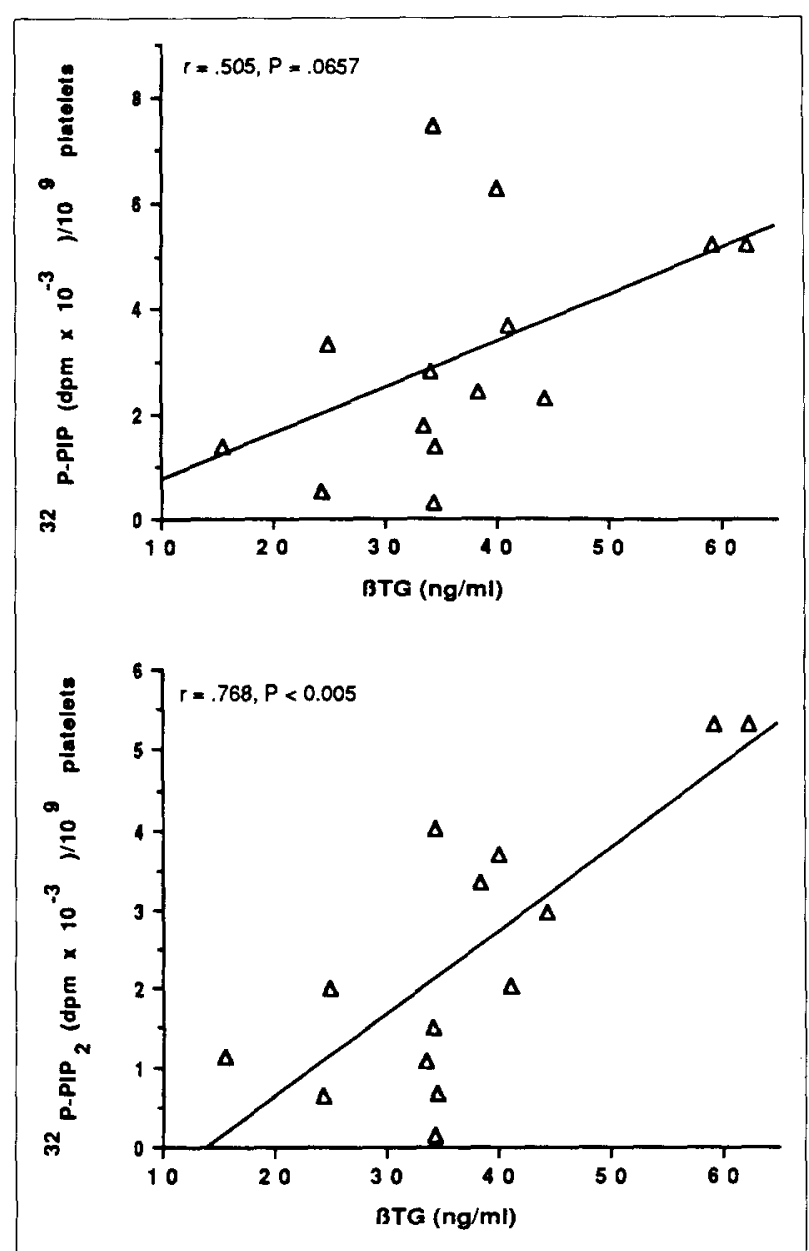

Figure 6. Relationship between in vivo platelet activation (de termined as plasma $\beta$-TBG) and ${ }^{32} \mathrm{P}_{i}$ incorporation into the platelet polyphosphoinositides. Top, ${ }^{32} \mathrm{P}$-PIP versus plasma $\beta$ TBG $(n=29)$, and bottom, ${ }^{32} \mathrm{P}-\mathrm{PIP} 2$ versus plasma $\beta$-TBG ( $=30$ ). Plasma $\beta$-TBG was determined from samples collected at the time platelets were obtained for phosphoinositide turnover and aggregation studies. The correlation coefficient for linear regression analysis $(r)$ and significance value $(p)$ are given. 
primary factor in platelet dysfunction and atherosclerosis.

Age-dependent changes in phosphoinositide content in rat pituitary have been reported [31]. Unlike the rat pituitary in which a decrease was reported [31] we found an increase in platelet membrane PIP and $\mathrm{PIP}_{2}$ with advancing age. Furthermore, we found that upon stimulation with thrombin, hydrolysis of $\mathrm{PIP}_{2}$ and generation of PA increase with advancing years of the individual. Since hydrolysis of $\mathrm{PIP}_{2}$ is known to accompany the initial changes in energy utilization as well as calcium mobilization in the platelet, it would be attractive to speculate that the increase in in vitro and in vivo platelet function accompanying aging might be

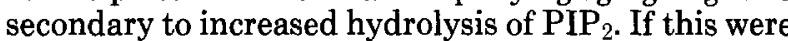
the case, changes in thromboxane formation or inhibition thereof might not impact on platelet function or the clinical outcome, as has been reported [22].

The striking increase in percent aggregatory responses to ADP from $10 \%$ to $40 \%$ indicates an enor mous variation in the normal aging population, and this does not include subjects over 62 years of age. The wide variability in these measurements dictates a need for age-dependent control values to be established before quantitative interpretation of data can be made outside of the research setting. Furthermore, it is not necessarily clear that the platelet changes are intrinsic to the platelet and contribute to vascular disease or simply reflect asymptomatic vascular disease not defined by our rigorous inclusion criteria [32]. Further research into these relationships is clearly necessary.

In summary, we have shown that platelet activity increases with age. Polyphosphoinositide content as measured by basal ${ }^{32} \mathrm{P}_{\mathrm{i}}$ incorporation into PIP and $\mathrm{PIP}_{2}$ also increases with age. The increase in polyphosphoinositide content correlated positively with both platelet aggregation and $\beta-T B G$ release and polyphosphoinositide hydrolysis. We conclude that the enhanced platelet activity found in the aging individual might be a consequence of increased platelet polyphosphoinositide turnover.

\section{ACKNOWLEDGMENT}

We wish to acknowledge the secretarial assistance of Lynde Amstutz, the expert statistical help from Morton Brown, and the technical assistance of Douglas Heady.

\section{REFERENCES}

1. Ross R: The pathogenesis of atherosclerosis — an update. N Engl J Med 1986 314: 488-496.

2. Johnson $M$, Ramey $E$, Ramwell $P$ : Sex and age differences in human platelet aggregation. Nature 1975; 253: 355-357.

3. Damad Study Research Group: Effect of age on ADP-induced platelet aggregation in diabetic and non-diabetic subjects. Thromb Res $1981 ; 22: 687-692$.

4. Kaplan KL, Owen J: Plasma levels of $\beta$-thromboglobulin and platelet factor 4 as indices of platelet activation in vivo. Blood 1981: 57: 199-202.

5. Lloyd JV, Nishizawa EE, Mustard JF: Effect of ADP-induced shape change on incorporation of $32 \mathrm{P}$ into platelet phosphatidic acid and mono-, di- and triphosphatidyl inositol. Br J Haematol 1973; 25: 77-99.

6. Vickers JD, Kinlough-Rathbone RL, Mustard JF: Changes in phosphatidylinositol 4,5-bisphosphate 10 seconds after stimulation of washed rabbit platelets with ADP. Blood 1982; 60: 1247-1250.

7. Billah MM, Lapetina EG: Rapid decrease of phosphatidylinositol 4,5-bisphosphate in thrombin-stimulated platelets. J Biol Chem 1982; 257: 12705-12708.

8. Berridge J: Inositol trisphosphate and diacylglycerol as second messengers. Biochem J 1984; 220: 345-360

9. Rink TJ, Sanchez A, Hallam TJ: Diacylglycerol and phorbol ester stimulate secre tion without raising cytoplasmic free calcium in human platelets. Nature 1983; 305: 317-319.

10. Nishizuka Y: Turnover of inositol phospholipids and signal transduction. Science 1984; 225: 1365-1368.

11. Born GVR, Cross MJ: The aggregation of blood platelets. J Physiol 1963; 168 : $178-195$.

12. Detwiler TC. Feinman RD: Kinetics of the thrombin-induced release of adenosine trisphosphate by platelets. Comparison with release of calcium. Biochemistry 1973; 12: 2462-2468.

13. Lane DA, Ireland $\mathrm{H}$, Wolff $\mathrm{S}$, Ranasinghe $\mathrm{E}$, Dawes J: Detection of enhanced in vivo platelet alpha-granule release in different patient groups-comparison of beta-thromboglobulin, platelet factor 4 , and thrombospondin assays. Thromb Haemost 1984; 52: 183-187.

14. Agranoff BW, Murthy P, Seguin EB: Thrombin-induced phosphodiesteratic cleavage of phosphatidylinositol bisphosphate in human platelets. J Biol Chem 1983; 258: 2076-2078

15. Jolles J, Zwiers H, Deffer A, Wirtz KWA, Gispend WH: Corticotropin-(1-24) tetracosapeptide affects protein phosphorylation and polyphosphoinositide metabolism in rat brain. Biochem $J 1981 ; 194$ : 283-291.

16. Duck-Chong C: A rapid sensitive method for determining phospholipid phosphorus involving digestion with magnesium nitrate. Lipids 1979: 14: 492-497. 17. Wilson DB, Neufeld EJ, Majerus PW: Phosphoinositide interconversion in thrombin stimulated human platelets. J Biol Chem 1985; 260: 1046-1051.

18. Bastyr EJ, Kadrofske MM, Vinik Al: Platelet aggregation and $32 \mathrm{P}$ incorporation into the phosphatidylinositols increases with age (abstr). Clin Res 1988; 36: 477A. 19. Sie P. Montagut J, Blanc M, et al: Evaluation of some platelet parameters in a group of elderly people. Thromb Haemost 1981: 45: 197-199.

20. Ludlam CA: Evidence for the platelet specificity of $\beta$-thromboglobulin and studies on its plasma concentration in healthy individuals. $\mathrm{Br} J$ Haematol 1979; 41: $271-278$

21. Zahavi J, Jones NAG, Leyton J, Dubiel M, Kakkar W: Enhanced in vivo platelet "release reaction" in old healthy individuals. Thromb Res 1980; 17: 329-336.

22. Bastyr EJ, Kadrofske MM, Dershimer RC, Vinik Al: Decreased platelet phosphoinositide turnover and enhanced platelet activation in IDDM. Diabetes 1989; 38: $1097-1102$

23. Lecrubier $C$, Scarabin PY, Grauso F, Samama M: Platelet aggregation related to age in diabetes mellitus. Haemostasis 1980; 9:43-51

24. Yamanishi J, Sano H, Saito K, Furuta F, Fukuzaki H: Plasma concentrations of platelet-specific proteins in different stages of essential hypertension: interactions between platelet aggregation, blood lipids and age. Thromb Haemost 1985; 54: 539-543

25. Prisco D, Rogasi PG, Matucci $M$, et al: Age-related changes in platelet lipid composition. Thromb Res 1986; 44: 427-437.

26. Stewart MJ, Gerrard JM, White JG: Effect of cholesterol on production of thromboxane $B_{2}$ by platelets in vitro. N Engl J Med 1980; 302: 6-10.

27. Tremoli $E$, Maderna P, Colli S, Morazzoni G, Sirtori M Sirtori CR Increased platelet sensitivity and thromboxane B2 formation in type-Il hyperlipoproteinaemic patients. Eur J Clin Invest 1984; 14: 329-333.

28. Vericel $E$, Lagarde M, Mendy F, Courpon PH, Dechavanne M: Effects of linoleic acid and gamma-linolenic acid intake on platelet functions in elderly people. Thromb Res 1986: 42: 499-509.

29. Siess W, Schere B, Altlung B, Roth B, Kurzmann I, Weber PC: Platelet membrane fatty acids, platelet aggregation, and thromboxane formation during mackerel diet. Lancet 1980; 4: 441-444.

30. Colwell JA, Bingham SF, Abraira C, et al, and The Cooperative Study Group: Veterans Administration Cooperative Study on antiplatelet agents in diabetic patients after amputation for gangrene: $\|$. Effects of aspirin and dipyridamole on atherosclerotic vascular disease rates. Diabetes Care 1986; 9: 140-148.

31. Bonetti AC, Bellini F, Calderini G, Galbiati E, Toffano G: Age-dependent changes in the mechanisms controlling prolactin secretion and phosphatidylinositol turnover in male rats: effect of phosphatidylserine. Neuroendocrinology 1987; 45 : $123-129$.

32. Levine SP: Secreted platelet proteins as markers for pathological disorders. In Phillips D, Shuman M, eds. Biochemistry of Platelets. New York: Academic Press 1986: 377-415. 